Conclusion Existing and new services must complete comprehensive evaluations to establish whether services are inclusive, accessible and address, rather than exacerbate, sexual health inequalities. Now is a critical time to identify the effectiveness of these digital health interventions given the wave of service expansions across the UK.

\section{P180 SELF-SAMPLING DEMONSTRATES COMPARABLE SENSITIVITY AND SPECIFICITY TO CLINICIAN-SAMPLING FOR HPV TESTING AMONG MSM IN CHINA}

${ }^{1} \mathrm{Y} \mathrm{Ni}{ }^{*},{ }^{1} \mathrm{Y} \mathrm{Lu},{ }^{2} \mathrm{X} \mathrm{He},{ }^{2} \mathrm{Y} \mathrm{Li},{ }^{1} \mathrm{C} \mathrm{Xu},{ }^{1} \mathrm{~W}$ Tang. ${ }^{1}$ University of North Carolina At Chapel Hill Project-China, Guangzhou, China; ${ }^{2}$ Zhuhai Xutong Voluntary Services Center, Zhuhai, China

10.1136/sextrans-2021-sti.275

Human papillomavirus (HPV) can cause genital warts and cervical, anal, and penile cancers. HPV self-sampling is an acceptable and feasible approach for HPV testing among women. Despite emerging research stated a higher risk of HPV infection among men who have sex with men (MSM), few guidelines are available for HPV self-sampling among MSM. Adding HPV self-sampling as a complement may benefit MSM and improve STI testing services among key populations. This study aimed to evaluate the feasibility and accuracy of HPV self-sampling among MSM.

MSM who were aged 18 or above, had sex with men in the past year were recruited. Eligible participants followed the instruction to self-collect specimens using oral fluid, penile, and rectal swab. Then a clinician or trained staff collected specimens from same areas. All specimens were processed using PCR test for 14 high-risk subtypes and 2 low-risk subtypes. PCR results were defined as the gold standard when assessing the performance of self-sampling and clinician-sampling. Sensitivity and specificity were calculated for each approach independently, and then chi-square test was used to compare two approaches.

A total of 211 MSM were recruited and tested from April to October 2020 in Zhuhai, China. The mean age of MSM was 31 years old $(\mathrm{SD}=7.9)$. The overall prevalence of HPV among participants was 49\% (103/211). Clinician-sampled specimens detected 91 of 103 MSM infected with HPV, with a sensitivity of $88.3 \%$ (95\% CI: 80.2-93.6), and specificity of $100.0 \% \quad$ (95\% CI: 95.7-100.0), respectively. Self-sampled specimens detected 81 of 103 MSM infected with HPV, with a sensitivity of $78.6 \%$ (95\% CI: 69.2-85.9), and a specificity of $100.0 \%$ (95\% CI: 95.7-100.0), respectively. The sensitivity was comparable between the clinician-sampling and self-sampling among MSM $(\mathrm{P}=0.09)$.

HPV self-sampling is feasible among MSM and it holds the potential in scaling-up HPV testing services among key populations.

\section{P181 PUBLIC HEALTH DECISION-MAKERS' PERSPECTIVES ON APPROACHES TO ECONOMIC EVALUATION FOR SEXUALLY TRANSMITTED INFECTION CONTROL PROGRAMMES}

'S Bloch*, 'L Jackson, 'E Frew, ${ }^{2} \mathrm{~J}$ Ross. 'University of Birmingham, Birmingham, UK; ${ }^{2}$ Whittall Street Clinic, University Hospitals Birmingham NHS Foundation Trust, Birmingham, UK

10.1136/sextrans-2021-sti.276
Background Economic evaluations aim to inform decision-makers about the cost-effectiveness of health interventions. However, currently economic evaluations may be underutilised by local public health decision-makers.

Whilst research has been conducted with public health decision-makers, the needs and priorities of those primarily concerned with sexual health services (SHS) have not been fully considered. This research aimed to gain an insight into how decision-makers based in England, responsible for SHS use economic evidence to inform decision-making.

Methods In-depth semi-structured interviews with participants who were purposefully sampled through a snowballing approach until saturation was reached. The interviews were transcribed ad-verbatim and analysed using the framework analysis method.

Results Fifteen qualitative interviews with 17 participants were conducted. Eight participants were local commissioners, four were national commissioners, and three were responsible for service provision. Three main themes were identified:

Context/commissioning - Decision-making processes around SHS were reported as complex and involving multiple stakeholders. Different services are commissioned by a wide-range of decision-makers, and the different types of contracting SHS, affect the comparability of provision.

Costs and budgets - Decision-makers described pressures on budgets due to increasing demand for SHS and funding limitations. Nearly all stated that the fragmentation of commissioning created issues around budget flow. Another significant issue was that savings were realised by different parts of the system to those who pay for them.

Using economic evidence - Participants mainly focussed on economic evidence in terms of return on investment. Although broader outcomes such as impacts on inequalities were seen as relevant, this was often overshadowed by cost and cost-saving concerns. Helpful evidence was described as being adaptable to the local population and including costs relevant to local areas.

Conclusions Future economic evaluations of SHS need to be tailored to ensure that they provide economic evidence that meets the needs of decision-makers.

\section{P182 IDENTIFYING THE PREP GAP: A SYSTEMATIC REVIEW EXPLORING EQUITY IN THE HIV-PREP CARE CONTINUUM IN HIGH INCOME COUNTRIES}

${ }^{1} \mathrm{M}$ Cabecinha*, ${ }^{2} \mathrm{D}$ Solomon, ${ }^{1} \mathrm{G}$ Rait, ${ }^{3,4} \mathrm{~J}$ Saunders, ${ }^{4,5} \mathrm{H}$ Mohammed. ${ }^{1}$ Research Department of Primary Care and Population Health, University College London, London, UK ${ }^{2}$ Institute for Global Health, University College London, London, UK; ${ }^{3}$ UCL Centre for Clinical Research in Infection and Sexual Health, Institute for Global Health, University College London, London, UK; ${ }^{4}$ Blood Safety, Hepatitis, Sexually Transmitted Infections (STI) and HIV Division, National Infection Service, Public Health England, London, UK; ${ }^{5}$ Infection and Population Health, Institute for Global Health, University College London, London, UK

\subsection{6/sextrans-2021-sti.277}

Background Equitable implementation of HIV Pre-Exposure Prophylaxis (PrEP) is not well defined, particularly for populations already experiencing high levels of health inequity (e.g. people experiencing poverty or other social disadvantages). The five stages of the PrEP care-continuum (PCC) (awareness, acceptability, uptake, adherence, retention) can help evaluate PrEP implementation, but the extent to which key characteristics that are important for health equity are considered 